\title{
Virtual Continuous CWmin Control Scheme of WLAN
}

\author{
Yuki SANGENYA $^{\dagger a)}$, Fumihiro INOUE ${ }^{\dagger}$, Student Members, Masahiro MORIKURA ${ }^{\dagger}$, Fellow \\ Koji YAMAMOTO $^{\dagger}$, Senior Member, Fusao NUNO ${ }^{\dagger \dagger}$, Member, and Takatoshi SUGIYAMA ${ }^{\dagger \dagger}$, Senior Member
}

\begin{abstract}
SUMMARY In this paper, a priority control problem between uplink and downlink flows in IEEE 802.11 wireless LANs is considered. The minimum contention window size (CWmin) has a nonnegative integer value. CWmin control scheme is one of the solutions for priority control to achieve the fairness between links. However, it has the problem that CWmin control scheme cannot achieve precise priority control when the $\mathrm{CWmin}$ values become small. As the solution of this problem, this paper proposes a new $\mathrm{CWmin}$ control method called a virtual continuous $\mathrm{CWmin}$ control (VCCC) scheme. The key concept of this method is that it involves the use of small and large CWmin values probabilistically. The proposed scheme realizes the expected value of CWmin as a nonnegative real number and solves the precise priority control problem. Moreover, we proposed a theoretical analysis model for the proposed VCCC scheme. Computer simulation results show that the proposed scheme improves the throughput performance and achieves fairness between the uplink and the downlink flows in an infrastructure mode of the IEEE 802.11 based wireless LAN Throughput of the proposed scheme is $31 \%$ higher than that of a conventional scheme when the number of wireless stations is 18 . The difference between the theoretical analysis results and computer simulation results of the throughput is within $1 \%$ when the number of STAs is less than 10 .

key words: CSMA/CA, IEEE 802.11 DCF, CWmin control, fairness, performance analysis model
\end{abstract}

\section{Introduction}

IEEE 802.11 distribution control function (DCF) is a carrier sense based medium access control (MAC) protocol defined by the IEEE 802.11 standard [1] and has been widely used in wireless local area networks (WLANs). A WLAN is one of the most promising candidates for constructing a home area network (HAN) connecting various home electronics and sensors.

In WLANs based on the IEEE 802.11 standard, each WLAN station (WS), including an access point (AP) and stations (STAs), has the same transmission opportunity [2]. Therefore, unfairness between the uplink and the downlink flows arises in networks wherein many STAs belong to a single AP [3]. As a solution to this problem, a minimum contention window size (CWmin) control has been studied [4]-[6].

Abeysekera et al. showed that fairness between the up-

Manuscript received March 16, 2013.

Manuscript revised July 18, 2013.

${ }^{\dagger}$ The authors are with the Graduate School of Informatics, Kyoto University, Kyoto-shi, 606-8501 Japan.

${ }^{\dagger}$ The authors are with the NTT Access Network Service Systems Laboratories, NTT Corporation, Yokosuka-shi, 239-0847 Japan.

a) E-mail: sangenya@imc.cce.i.kyoto-u.ac.jp

DOI: $10.1587 /$ transfun.E97.A.40 link and the downlink flows can be achieved and that the quality of service $(\mathrm{QoS})$ requirements are guaranteed by optimizing the CWmin value at the AP in a network operating in the infrastructure mode of IEEE 802.11 [6]. However, the conventional CWmin control cannot work accurately subject to the constraint that the downlink throughput be equal to the total uplink throughput because CWmin is in the range of a nonnegative integer [7].

This paper proposes a virtual continuous CWmin control (VCCC) scheme that can virtually realize the CWmin value as a nonnegative real number. The proposed VCCC scheme enables accurate control under the constraint that the downlink throughput be equal to the total uplink throughput. Moreover, a theoretical analysis model for the proposed VCCC scheme is proposed. The results obtained from the theoretical analysis are in good agreement with those obtained from computer simulations when the number of STAs is less than 10 .

The rest of this paper is organized as follows. The system models are presented in Sect. 2. The conventional scheme is described in Sect.3. In Sect.4, the proposed VCCC scheme is presented. In Sect. 5, we show the numerical results of the conventional and proposed schemes. Finally, we present some concluding remarks in Sect. 6.

\section{System Model}

Let us consider a single-hop WLAN network shown in Fig. 1. An AP and $n$ STAs are deployed in the network. The AP is connected by wired networks and serves as a gateway, and the STAs belong to the AP in an infrastructure mode. In the MAC layer, the carrier sense multiple access with collision avoidance (CSMA/CA) protocol based on the IEEE 802.11 standard is used. It is assumed that the capture ef-

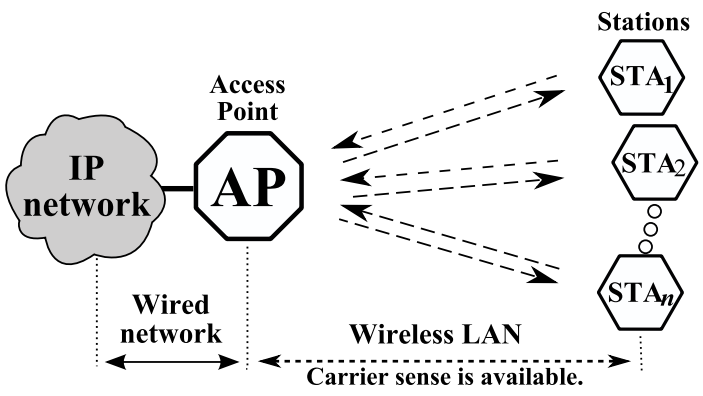

Fig. 1 Single-hop WLAN network with infrastructure mode. 
Table 1 Major parameters of 802.11a standard.

\begin{tabular}{c|c}
\hline PHY data rate & $6,9,12,18,24,36,48,54 \mathrm{Mbit} / \mathrm{s}$ \\
MAC header & $26 \mathrm{byte}$ \\
FCS & $4 \mathrm{byte}$ \\
PHY header & $20 \mu \mathrm{s}$ \\
Tail bit & $6 \mathrm{bit}$ \\
Service bit & $16 \mathrm{bit}$ \\
ACK length & $20 \mu \mathrm{s}+134 \mathrm{bit}$ \\
SIFS & $16 \mu \mathrm{s}$ \\
DIFS & $34 \mu \mathrm{s}$ \\
Slot time & $9 \mu \mathrm{s}$ \\
OFDM symbol time & $4 \mu \mathrm{s}$ \\
CWmin & 15 \\
CWmax & 1023 \\
Maximum backoff stage & 6 \\
Short retry limit & 7 \\
\hline
\end{tabular}

fect does not occur, i.e., no WSs can obtain information at the instant a frame collision occurs. In this paper, we assume saturated traffic load conditions for simplicity: that is, the transmission queues of the AP and STAs are always nonempty. The propagation delay of each WS is negligible. In order to evaluate the throughput performance in the MAC layer, it is assumed that all the WSs generate user datagram protocol (UDP) traffic. We consider a WLAN based on the IEEE 802.11a standard, the parameters of which are listed in Table 1. We define an unidirectional throughput as

$$
\min \left\{S_{\text {up }}, S_{\text {down }}\right\} \text {, }
$$

where $S_{\text {up }}$ and $S_{\text {down }}$ denote the uplink and downlink throughput, respectively. The fairness between the uplink and the downlink flows can be achieved by maximizing the unidirectional throughput. In this paper, the purpose of $\mathrm{CWmin}$ control is to maximize the unidirectional throughput, and it is considered that the system is optimized when the unidirectional throughput is maximized.

\section{Conventional Scheme}

The conventional dynamic CWmin control (DCC) scheme [6] and its problems are described in this section. In the conventional DCC scheme, to solve an unfairness problem between the uplink and the downlink flows, the CWmin value of the AP is determined according to the number of STAs that belong to a single AP. The backoff period when the WS waits before the frame transmission is depends on each WS's CWmin value. The frame transmission opportunity of the WS increases with a decrease in the WS's CWmin value. The CWmin values of the AP and the STA are expressed as $\mathrm{CWmin}_{\mathrm{AP}}$ and $\mathrm{CWmin}$ STA, respectively. The fairness between the uplink and the downlink flows can be achieved when the AP has the same transmission opportunity as all the STAs that belong to the AP. This is achieved by deriving the optimum $\mathrm{CWmin}_{\mathrm{AP}}$ value on the basis of $\mathrm{CWmin}_{\mathrm{STA}}$ value and other parameters. To evaluate the numerical value of the optimum $\mathrm{CWmin}$ AP, the default value of $\mathrm{CWmin}$ STA that is defined in the IEEE 802.11 standard was used [6].

Figure 2 shows the throughputs with the conventional $\mathrm{CWmin}$ control according to $\mathrm{CWmin} \mathrm{AP}_{\mathrm{AP}}$ value when

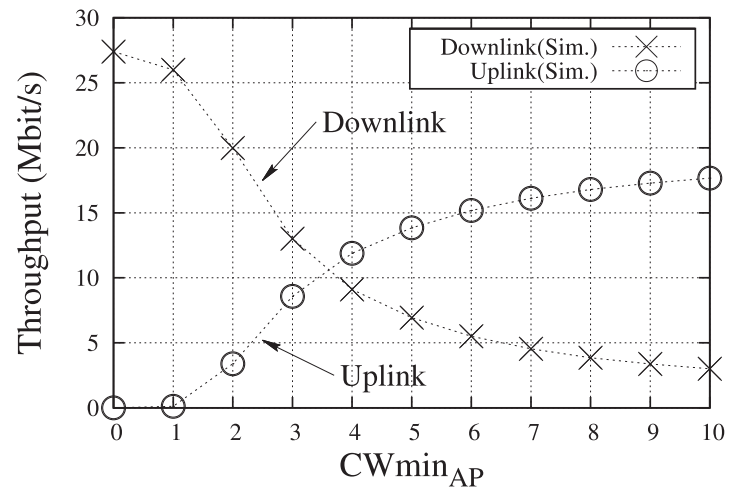

Fig. 2 Throughputs with conventional CWmin control (Number of STAs $n: 10$, PHY data rate: $36 \mathrm{Mbit} / \mathrm{s}$ ).

the number of STAs is 10 . The downlink throughput $S_{\text {down }}$ decreases and the uplink throughput $S_{\text {up }}$ increases monotonously with an increase in $\mathrm{CWmin}$ AP value. The unidirectional throughput can be maximized if $\mathrm{CWmin}_{\mathrm{AP}}$ value is set between three and four, as shown in Fig. 2. However, $\mathrm{CWmin}_{\mathrm{AP}}$ value should be an integer. Thus, according to Fig. 2, the optimized CWmin ${ }_{\mathrm{AP}}$ is four. If $\mathrm{CWmin}_{\mathrm{AP}}$ value can be a real number, we can control the unidirectional throughput more precisely.

\section{Proposed Scheme}

In this section, the new CWmin control method (VCCC scheme) that can virtually realize CWmin value as a nonnegative real number is proposed.

\subsection{Virtual Continuous CWmin Control (VCCC)}

An AP that employs the basic access mechanism of IEEE 802.11 DCF resets its contention window size when the frame transmission is successful or the retry limit is reached. In the case of the legacy DCF, the CW value is reset at $l$, which has a nonnegative integer value.

On the other hand, CW is determined probabilistically as follows in the proposed VCCC scheme. The proposed method is applied to the AP.

$$
\mathrm{CWmin}_{\mathrm{AP}}= \begin{cases}l & \left(\text { Probability of } \alpha_{\mathrm{HP}}\right), \\ l+1 & \left(\text { Probability of } 1-\alpha_{\mathrm{HP}}\right),\end{cases}
$$

where $\alpha_{\mathrm{HP}}$ that is in the range of $0<\alpha_{\mathrm{HP}} \leq 1$, is the probability that $C W \min _{\mathrm{AP}}$ value becomes $l$. Figure 3 shows the procedure of the proposed VCCC scheme. Once an AP sets $\mathrm{CWmin}_{\mathrm{AP}}$ value to $l$ or $l+1$, the $\mathrm{AP}$ uses the same $\mathrm{CWmin}$ AP value until it resets its $\mathrm{CW}$ next time after a successful transmission or reaching the retry limit. The AP of the proposed VCCC scheme has two states and repeats them probabilistically. If the AP set CWmin ${ }_{\mathrm{AP}}$ value to small value $l$, the AP has a high priority state (HP). If the AP set $\mathrm{CWmin}$ AP value to large value $l+1$, the AP has a low priority state (LP). The expected value of CWmin ${ }_{A P}$ with the proposed VCCC scheme is expressed as 


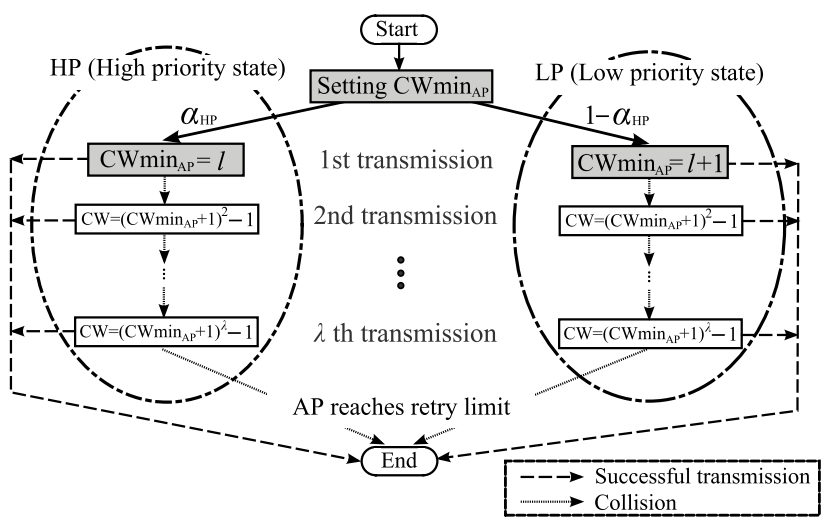

Fig. 3 Procedure of the virtual continuous CWmin control.

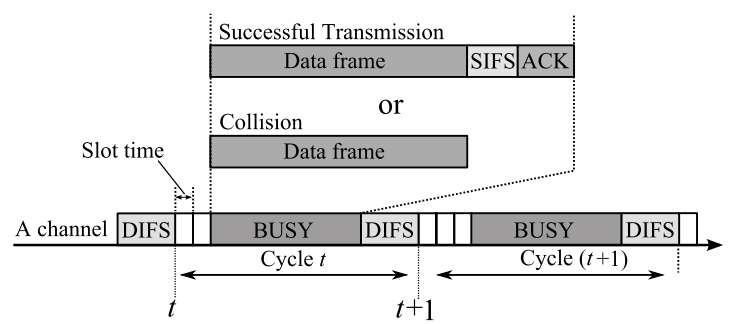

Fig. 4 The channel access of IEEE 802.11 DCF and the definition of the channel access cycle.

$$
\begin{aligned}
E\left[\mathrm{CWmin}_{\mathrm{AP}}\right] & =l \cdot \alpha_{\mathrm{HP}}+(l+1)\left(1-\alpha_{\mathrm{HP}}\right) \\
& =l-\alpha_{\mathrm{HP}}+1 .
\end{aligned}
$$

When we determine the expected value of $\mathrm{CWmin}_{\mathrm{AP}}$, which is a nonnegative real number, we derive the value of $l$ that is given by the following equation:

$$
l=\left\lfloor E\left[\mathrm{CWmin}_{\mathrm{AP}}\right]\right\rfloor,
$$

where $\lfloor z\rfloor$ stands for the maximum integer that is not greater than $z$ which is a real number. The value of $\alpha_{\mathrm{HP}}$ is determined by Eq. (3) when $l$ and $E\left[C W \min _{\mathrm{AP}}\right]$ are given.

\subsection{Theoretical Analysis}

Tinnirello et al. proposed an accurate analytical model of IEEE 802.11e enhanced distributed channel access (EDCA) [8]. In this section, we propose an analytical model, which is expanded from Tinnirello's model and can express the throughput performance of the proposed VCCC scheme.

\subsubsection{Analytical Model of IEEE 802.11}

Figure 4 shows an example of the IEEE 802.11 DCF and the cycle introduced in [8]. The channel access of the IEEE 802.11 DCF is considered as a repetition of a cycle. A cycle starts from the end of a DIFS period and terminates at the end of the next DIFS. Each cycle consists of an initial random waiting time plus one transmission event or a frame collision event. A discrete-time $t$ denotes the start of a $t$ th cycle.
Let $x(t)$ be the duration of the initial random time in cycle $t$ expressed in number of backoff slots. A saturated traffic load condition is assumed, and all the WSs compete persistently under that condition. $x(t)$ is expressed as

$$
x(t)=\min _{\Phi}\left(b_{\Phi}(t)\right)
$$

where $b_{\Phi}(t)$ is the backoff counter value of $\mathrm{WS}_{\Phi}$ at time t. The $\mathrm{WS}_{\Phi}$ is considered as AP_HP, AP_LP, or STA in the case of the proposed VCCC scheme. As described in Fig. 3, AP_HP and AP_LP represent as the AP with the HP and LP states, respectively. Tagged station index $k$ is used in [8]; however, we use the WS index $\Phi$ in place of index $k$. The transmission event occurring in cycle $t$ depends on the natural number $y$, which is the number of WSs whose backoff counter value is $x(t)$. Successful transmission event occurs if $y=1$, and the frame collision event occurs if $y>1$. Thus, we can know the event occurring in the cycle $t$ if $b_{\Phi}(t)$ is obtained.

Let $B_{\Phi}(j)$ be the steady-state distribution of the $\mathrm{WS}_{\Phi}$ 's backoff counter. $B_{\Phi}(j)$ is defined as

$$
B_{\Phi}(j)=\lim _{t \rightarrow \infty} \operatorname{Pr}\left\{b_{\Phi}(t)=j\right\} .
$$

The existence of such a steady-state distribution is described in [8]. $B_{\Phi}(j)$ depends on the $\mathrm{WS}_{\Phi}$ 's parameters, such as CWmin and CWmax values.

\subsubsection{Definition of Variables}

$\mathrm{WS}_{\Phi}$ has the following set of MAC parameters:

- $R_{\Phi}$ : Retry limit of $\mathrm{WS}_{\Phi}$.

- $m_{\Phi}$ : Maximum number of retransmissions until a frame is discarded. Thus, $m_{\Phi}$ is $R_{\Phi}-1$.

- $W_{\Phi, s}: \mathrm{CW}$ value used in backoff stage of $s$.

- $W_{\Phi, \max }: \mathrm{CWmax}$ value of $\mathrm{WS}_{\Phi}$.

- $s_{\Phi}(t)$ : Backoff stage value of $\mathrm{WS}_{\Phi}$ when DIFS duration ends. $s_{\Phi}(t)$ has a value in the range of $\left[0, m_{\Phi}\right]$.

- $b_{\Phi}(t)$ : Backoff counter value of $\mathrm{WS}_{\Phi}$ after DIFS duration. $b_{\Phi}(t)$ is randomly selected from the range of $\left[0, W_{\Phi, s}\right]$.

Let $\Pi_{\Phi}(s, j)$ be a distribution such as

$$
\Pi_{\Phi}(s, j)=\lim _{t \rightarrow \infty} \operatorname{Pr}\left\{s_{\Phi}(t)=s, b_{\Phi}(t)=j\right\} .
$$

$\Pi_{\Phi}(s, j)$ denotes the probability that the $\mathrm{WS}_{\Phi}$ has a backoff stage value of $s \in\left[0, m_{\Phi}\right]$ and a backoff counter value of $j \in\left[0, W_{\Phi, \max }\right]$ in the steady-state cycle. By using $\Pi_{\Phi}(s, j)$, $B_{\Phi}(j)$ defined in Eq. (6) is expressed as

$$
B_{\Phi}(j)=\sum_{s=0}^{m_{\Phi}} \Pi_{\Phi}(s, j) .
$$

Let us focus on $\mathrm{WS}_{\Phi}$. Two probabilities that determine the states of other competing WSs are defined as follows.

- $Q_{\Phi}(i)$ : The probability that no competing WSs transmit 
before the occurrence of the slot index $i$. Slot index $i$ is defined as the $i$ th slot time after the DIFS period. $i$ is greater than or equal to zero.

- $T_{\Phi}(i)$ : The probability that the transmission of the other competing WSs occurs in a cycle exactly at slot index $i$.

It can be known that if $\mathrm{WS}_{\Phi}$ 's transmission succeeds or fails according to the probabilities of $Q_{\Phi}(\cdot)$ and $T_{\Phi}(\cdot)$. The cumulative distribution $\beta_{\Phi}(i)$ is defined as follows to obtain $Q_{\Phi}(i)$.

$$
\beta_{\Phi}(i)= \begin{cases}0 & (i \leq 0), \\ \sum_{j=0}^{i-1} B_{\Phi}(j) & (i>0) .\end{cases}
$$

$\beta_{\Phi}(i)$ represents the probability that $\mathrm{WS}_{\Phi}$ transmits a frame before the occurrence of slot index $i$. By using $\beta_{\Phi}(i), Q_{\Phi}(i)$ is calculated as

$$
\left\{\begin{array}{l}
Q_{\mathrm{AP} \_\mathrm{HP}}(i)=Q_{\mathrm{AP} \_\mathrm{PP}}(i)=\left\{1-\beta_{\mathrm{STA}}(i)\right\}^{n}, \\
Q_{\mathrm{STA}}(i)=\left[1-\left\{\beta_{\mathrm{AP} \_\mathrm{HP}}(i)+\beta_{\mathrm{AP} \_\mathrm{PP}}(i)\right\}\right]\left\{1-\beta_{\mathrm{STA}}(i)\right\}^{n-1},
\end{array}\right.
$$

where $Q_{\Phi}(0)=1$. By using $Q_{\Phi}(i), T_{\Phi}(i)$ is calculated as

$$
T_{\Phi}(i)=Q_{\Phi}(i)-Q_{\Phi}(i+1) .
$$

Note that the probabilities defined above can be expressed by using $\Pi_{\Phi}(s, j)$.

\subsubsection{Event Formularization with Proposed Scheme}

The proposed VCCC scheme can realize the $E[C W m i n]$ value as a nonnegative real number by changing the $\mathrm{CWmin}$ values probabilistically. The system proposed by Tinnirello can be used when CWmin value has a fixed value. In this section, we propose the modified system that can be applied to the proposed VCCC scheme.

The state transition probability between $\left\{s_{\Phi}(t), b_{\Phi}(t)\right\}$ and $\left\{s_{\Phi}(t+1), b_{\Phi}(t+1)\right\}$ depends on the occurrence of the four following cases.

case 1) The backoff counter of WS $S_{\Phi}$ resumes. It does not reach zero because of a transmission or collision event by the other WSs.

Let the previous state of $\mathrm{WS}_{\Phi}$ be $\left\{s_{\Phi}(t)=s, b_{\Phi}(t)=\right.$ $b+r\}$, where $r \in\left[0, W_{\Phi, s}-b\right]$. In this case, the backoff counter value of the $\mathrm{WS}_{\Phi}$ will be decremented by $r$, but it will not reach zero before cycle $t$ ends. The probability which $\mathrm{WS}_{\Phi}$ ends in state $\left\{s_{\Phi}(t+1)=s, b_{\Phi}(t+1)=b\right\}$ is

$$
T_{\Phi}(r) .
$$

Thus, $\Pi_{\Phi, \mathrm{BO}}(s, j)$, which denotes the contribution of case 1 to $\Pi_{\Phi}(s, j)$, is expressed as follows,

$$
\Pi_{\Phi, \mathrm{BO}}(s, j)=\sum_{r=0}^{W_{\Phi, s}-j} \Pi_{\Phi}(s, j+r) T_{\Phi}(r)
$$

case 2) The cycle ends with a collision event involving $W S_{\Phi}$, while $W S_{\Phi}$ has not yet reached its retry limit of $R_{\Phi}$.

In this case, the backoff stage value of $\mathrm{WS}_{\Phi}$ is incremented. If the previous state is $\left\{s_{\Phi}(t)=s-1, b_{\Phi}(t)=\right.$ $j$ \}, with $s-1<m_{\Phi}$, the transition probability to state $\left\{s_{\Phi}(t+1)=s, b_{\Phi}(t+1)=b\right\}$ is given by

$$
\frac{1}{W_{\Phi, s}+1} T_{\Phi}(j) \text {. }
$$

$\Pi_{\Phi, \mathrm{US}}(s, j)$, which denotes the contribution of case 2 to $\Pi_{\Phi}(s, j)$, is expressed as follows,

$$
\Pi_{\Phi, \mathrm{US}}(s, j)=\frac{1}{W_{\Phi, s}+1} \sum_{i=0}^{W_{\Phi, s-1}} \Pi_{\Phi}(s-1, i) T_{\Phi}(i) .
$$

case 3) The cycle ends with a collision event involving $W S_{\Phi}$, while $W S_{\Phi}$ has reached its retry limit of $R_{\Phi}$. In this case, the frame is dropped, a new frame is scheduled for the next transmission, and the backoff stage value is set to zero. For the previous state $\left\{s_{\Phi}(t)=m_{\Phi}, b_{\Phi}(t)=j\right\}$, the transition probability to state $\left\{s_{\Phi}(t+1)=0, b_{\Phi}(t+1)=b\right\}$ is

$$
\frac{1}{W_{\Phi, 0}+1} T_{\Phi}(j) \text {. }
$$

Let us consider $\Pi_{\Phi, \mathrm{RL}}(s, j)$, which denotes a contribution of case 3 to $\Pi_{\Phi}(s, j)$. In the case $3, \mathrm{WS}_{\Phi}$ resets its $\mathrm{CW} . \Pi_{\mathrm{STA}, \mathrm{RL}}(s, j)$ denotes the contribution of case 3 to $\Pi_{\mathrm{STA}}(s, j)$ and is expressed as

$$
\begin{aligned}
& \Pi_{\mathrm{STA}, \mathrm{RL}}(s, j) \\
& =\frac{1}{W_{\mathrm{STA}, 0}+1} \sum_{i=0}^{W_{\text {STA,max }}} \Pi_{\mathrm{STA}}\left(m_{\mathrm{STA}}, i\right) T_{\mathrm{STA}}(i) .
\end{aligned}
$$

On the other hand, an AP is applied to the proposed VCCC scheme, and it resets its CW according to the procedure shown in Fig. 3. Considering that the AP occupies the AP_HP state with the probability of $\alpha_{\mathrm{HP}}$, $\Pi_{\mathrm{AP} \_\mathrm{HP}, \mathrm{RL}}(s, j)$, which denotes the contribution of case 3 to $\Pi_{\mathrm{AP} \_\mathrm{HP}}(s, j)$, is expressed as

$$
\begin{aligned}
& \Pi_{\mathrm{AP} \_\mathrm{HP}, \mathrm{RL}}(s, j) \\
& =\frac{\alpha_{\mathrm{HP}}}{W_{\mathrm{AP} \_\mathrm{HP}, 0}+1} \sum_{\Psi} \sum_{i=0}^{W_{\Psi, \max }} \Pi_{\Psi}\left(m_{\Psi}, i\right) T_{\Psi}(i), \\
& \text { where } \Psi \in \mathrm{AP} \_\mathrm{HP}, \mathrm{AP} \_\mathrm{LP} .
\end{aligned}
$$

Considering that the AP occupies the AP_LP state with a probability of $1-\alpha_{\mathrm{HP}}, \Pi_{\mathrm{AP} \_\mathrm{LP}, \mathrm{RL}}(s, j)$ which denotes the contribution of case 3 to $\Pi_{\mathrm{AP} \_L \mathrm{P}}(s, j)$, is expressed as

$$
\Pi_{\mathrm{AP} \_\mathrm{LP}, \mathrm{RL}}(s, j)
$$




$$
\begin{aligned}
& =\frac{1-\alpha_{\mathrm{HP}}}{W_{\mathrm{AP} \_L P, 0}+1} \sum_{\Psi} \sum_{i=0}^{W_{\Psi, \max }} \Pi_{\Psi}\left(m_{\Psi}, i\right) T_{\Psi}(i), \\
& \text { where } \Psi \in \text { AP_HP, AP_LP. }
\end{aligned}
$$

case 4) The cycle ends with a successful transmission of $W S_{\Phi}$.

In this case, a transmission is successful, a new frame is scheduled for next transmission, and the backoff stage value is set to zero. Given the previous state $\left\{s_{\Phi}(t)=\right.$ $\left.s, b_{\Phi}(t)=j\right\}$, the transition probability to state $\left\{s_{\Phi}(t+\right.$ $\left.1)=0, b_{\Phi}(t+1)=b\right\}$ is

$$
\frac{1}{W_{\Phi, 0}+1} Q_{\Phi}(j+1) \text {. }
$$

Let us consider probability $\Pi_{\Phi, \mathrm{ST}}(s, j)$, which denotes the contribution of case 4 to $\Pi_{\Phi}(s, j)$. In case $4, \mathrm{WS}_{\Phi}$ resets its $\mathrm{CW}$. $\Pi_{\mathrm{STA}, \mathrm{ST}}(s, j)$, which denotes a contribution of case 4 to $\Pi_{\mathrm{STA}}(s, j)$, is expressed as

$$
\begin{aligned}
& \Pi_{\mathrm{STA}, \mathrm{ST}}(s, j) \\
& =\frac{1}{W_{\mathrm{STA}, 0}+1} \sum_{s=0}^{m_{\mathrm{STA}}} \sum_{i=0}^{W_{\mathrm{STA}, \max }} \Pi_{\mathrm{STA}}(s, i) Q_{\mathrm{STA}}(i+1) .
\end{aligned}
$$

On the other hand, the AP employs proposed VCCC scheme and it resets its $\mathrm{CW}$ according to the procedure shown in Fig. 3. Considering that the AP occupies the AP_HP state with the probability of $\alpha_{\mathrm{HP}}$, $\Pi_{\mathrm{AP} \_\mathrm{HP}, \mathrm{ST}}(s, j)$ which denotes the contribution of case 4 to $\Pi_{\mathrm{AP} \_\mathrm{HP}}(s, j)$, is expressed as

$$
\begin{aligned}
& \Pi_{\mathrm{AP} \_\mathrm{HP}, \mathrm{ST}}(s, j) \\
& =\frac{\alpha_{\mathrm{HP}}}{W_{\mathrm{AP} \_\mathrm{HP}, 0}+1} \sum_{\Psi} \sum_{s=0}^{m_{\Psi}} \sum_{i=0}^{W_{\Psi, \max }} \Pi_{\Psi}(s, i) Q_{\Psi}(i+1), \\
& \text { where } \Psi \in \mathrm{AP} \_\mathrm{HP}, \mathrm{AP} \_L P .
\end{aligned}
$$

Considering that the AP becomes the AP_LP state with the probability of $1-\alpha_{\mathrm{HP}}, \Pi_{\mathrm{AP} \_\mathrm{LP}, \mathrm{ST}}(s, j)$, which denotes the contribution of case 4 to $\Pi_{\mathrm{AP} \_L P}(s, j)$, is expressed as

$$
\begin{aligned}
& \Pi_{\mathrm{AP} \_\mathrm{LP}, \mathrm{ST}}(s, j) \\
& =\frac{1-\alpha_{\mathrm{HP}}}{W_{\mathrm{AP} \_\mathrm{LP}, 0}+1} \sum_{\Psi} \sum_{s=0}^{m_{\Psi}} \sum_{i=0}^{W_{\Psi, \max }} \Pi_{\Psi}(s, i) Q_{\Psi}(i+1), \\
& \text { where } \Psi \in \mathrm{AP} \_\mathrm{HP}, \mathrm{AP} \_L P .
\end{aligned}
$$

By considering all of the above cases, the steady-state distribution of $\Pi_{\Phi}(s, j)$ can be computed by solving and normalizing the following equations:

a) For $s=0, j=0$ (cases 3 and 4$)$ :

$$
\Pi_{\Phi}(0,0)=\Pi_{\Phi, \mathrm{RL}}(0,0)+\Pi_{\Phi, \mathrm{ST}}(0,0) .
$$

b) For $s=0,1 \leq j \leq W_{\Phi, 0}($ cases 1,3 and 4):

$$
\begin{aligned}
& \Pi_{\Phi}(0, j) \\
& \quad=\Pi_{\Phi, \mathrm{BO}}(0, j)+\Pi_{\Phi, \mathrm{RL}}(0, j)+\Pi_{\Phi, \mathrm{ST}}(0, j) .
\end{aligned}
$$

c) For $1 \leq s \leq m_{\Phi}, j=0$ (case 2$)$ :

$$
\Pi_{\Phi}(s, 0)=\Pi_{\Phi, \mathrm{US}}(s, 0) .
$$

d) For $1 \leq s \leq m_{\Phi}, 1 \leq j \leq W_{\Phi, \max }($ cases 1 and 2):

$$
\Pi_{\Phi}(s, j)=\Pi_{\Phi, \mathrm{BO}}(s, j)+\Pi_{\Phi, \mathrm{US}}(s, j) .
$$

On the other hand, the summation of the state probabilities for all WSs equals one. Thus, the following equations are given.

$$
\begin{aligned}
& \sum_{\Psi} \sum_{s=0}^{m_{\Psi}} \sum_{j=0}^{W_{\Psi}} \Pi_{\Psi}(s, j)=1, \\
& \text { where } \Psi \in \text { AP_HP, AP_LP. } \\
& \sum_{s=0}^{m_{\text {STA }}} \sum_{j=0}^{W_{\text {STA,max }}} \Pi_{\mathrm{STA}}(s, j)=1 .
\end{aligned}
$$

The system that is expressed as Eqs. (24)-(29) can be solved by using a fix-point iteration similar to that presented in [8]. We use the function " $f$ solve" in the MATLAB [9] to solve these equations. The solutions of $\Pi_{\mathrm{AP} \_\mathrm{HP}}(s, j), \Pi_{\mathrm{AP} \_\mathrm{LP}}(s, j)$, and $\Pi_{\mathrm{STA}}(s, j)$ are iteratively obtained. $B_{\Phi}(\cdot), \beta_{\Phi}(\cdot), Q_{\Phi}(\cdot)$, and $T_{\Phi}(\cdot)$ are derived from Eq. (8), Eq. (9), Eq. (10), and Eq. (11), respectively using the solutions of $\Pi_{\Phi}(s, j)$.

\subsubsection{Calculation of Throughput}

This section explains how to calculate the throughput of the proposed model. Let $P_{\text {succ }}(\Phi)$ be the probability that $\mathrm{WS}_{\Phi}$ 's transmission succeeds in the cycle, i.e., only $\mathrm{WS}_{\Phi}$ can transmit a data-frame in the cycle and $P_{\text {succ }}(\Phi)$ is derived as

$$
P_{\text {succ }}(\Phi)=\sum_{i=0}^{W_{\Phi, \max }} B_{\Phi}(i) Q_{\Phi}(i+1) .
$$

The probability $P_{\text {succ }}$ that success transmission occurs in the cycle is expressed as

$$
P_{\text {succ }}=n \cdot P_{\text {succ }}(\mathrm{STA})+P_{\text {succ }}\left(\mathrm{AP} \_ \text {HP }\right)+P_{\text {succ }}\left(\mathrm{AP} \_ \text {LP }\right) .
$$

Following variables are defined as:

- $E[x]$ : Expected value of the number of backoff slot in one cycle.

- $\sigma$ : Slot time.

- $T_{\text {succ }}$ : Duration of a successful transmission event.

- $T_{\text {coll }}$ : Duration of a collision event.

The expected value of one cycle duration is calculated as

$$
E[\text { cycle }]=E[x] \sigma+P_{\text {succ }} T_{\text {succ }}+\left(1-P_{\text {succ }}\right) T_{\text {coll }} \text {. }
$$

Let $Q_{0}(j)$ be the probability that no WSs transmit before the occurrence of backoff slot $j$ and it is computed as 


$$
Q_{0}(j)=\left[1-\left\{\beta_{\mathrm{AP} \_\mathrm{HP}}(j)+\beta_{\mathrm{AP} \_\mathrm{LP}}(j)\right\}\right]\left\{1-\beta_{\mathrm{STA}}(j)\right\}^{n} .
$$

The probability that the transmission event occurs in a cycle exactly at slot index $j$ is

$$
T_{0}(j)=Q_{\Phi}(j)-Q_{\Phi}(j+1) .
$$

On the other hand, $T_{0}(j)$ is the probability that there are $j$ backoff slots. Thus, the expected value of the number of backoff slot in one cycle is calculated as

$$
E[x]=\sum_{j=0}^{L} j \cdot T_{0}(j),
$$

where $L$ is the minimum of CWmax value in the WSs. As stated before, the uplink throughput $S_{\text {up }}$ and downlink throughput $S_{\text {down }}$ are computed as

$$
\begin{aligned}
& S_{\text {up }}=\frac{n \cdot P_{\text {succ }}(\mathrm{STA}) \cdot \text { PSIZE }}{E[\text { cycle }]}, \\
& S_{\text {down }}=\frac{\left\{P_{\text {succ }}(\text { AP_HP })+P_{\text {succ }}(\text { AP_LP })\right\} \cdot \text { PSIZE }}{E[\text { cycle }]},
\end{aligned}
$$

respectively, where PSIZE denotes the MAC service data unit (MSDU) size.

\section{Numerical Results}

\subsection{Single-rate and saturated traffic condition}

In this section, the computer simulation and theoretical analysis results are presented. We assume a saturated traffic load condition. It is assumed that $\mathrm{CWmin}_{\mathrm{STA}}$ value is 15 , which is the default value in the IEEE 802.11a standard [1]; the MSDU size of a data frame is 1500 bytes, and the PHY data rate is $36 \mathrm{Mbit} / \mathrm{s}$ in the network as shown Fig. 1. The basic access mechanism is used whenever a WS transmits a data frame. To evaluate the throughput performance, we employ a simulation platform on a Monte Carlo simulation using the $\mathrm{C}$ language. We confirmed that the simulation results of our program are in good agreement with those of QualNet 5.0 [10] under the same conditions explained previously with the conventional scheme described in Sect. 2.

In this study, the purpose of the CWmin control is to maximize the unidirectional throughput to achieve fairness.

Figures 5 and 6 show the throughput performances versus $E\left[C W \min _{\mathrm{AP}}\right]$ using the proposed VCCC scheme in the case where 10 and 20 STAs exist, respectively. In the figures, the curved lines represent the throughputs calculated by the theoretical analysis, and the symbols denote those obtained from the computer simulation. In the proposed scheme, the value of $l$ is given by Eq. (4). It increases in accordance with an increase in $E\left[C W \min _{\mathrm{AP}}\right]$ whose range and step size are $[0,10]$ and 0.01 , respectively in Figs. 5, 6 . Then the value of $\alpha_{\mathrm{HP}}$ is calculated by Eq. (3). These parameters give us the theoretical throughput of each link by Eqs. (36) and (37). Also they give us the simulation results

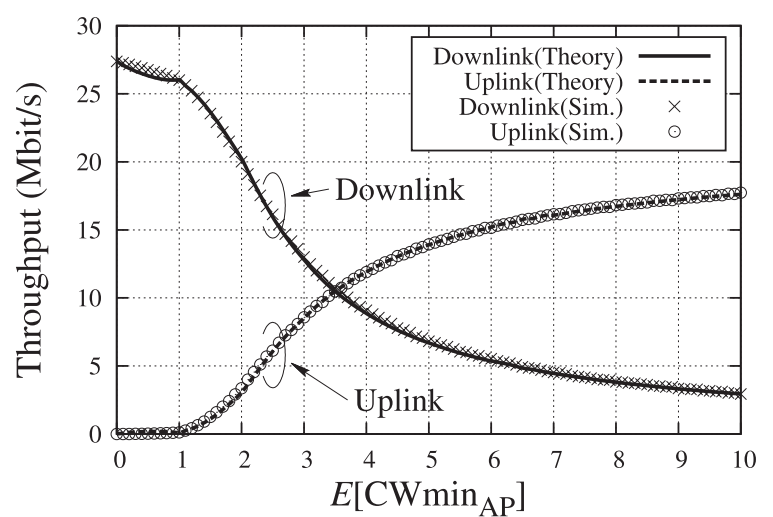

Fig. 5 Throughputs of the proposed scheme (Number of STAs $n: 10$, PHY data rate : $36 \mathrm{Mbit} / \mathrm{s}$ ).

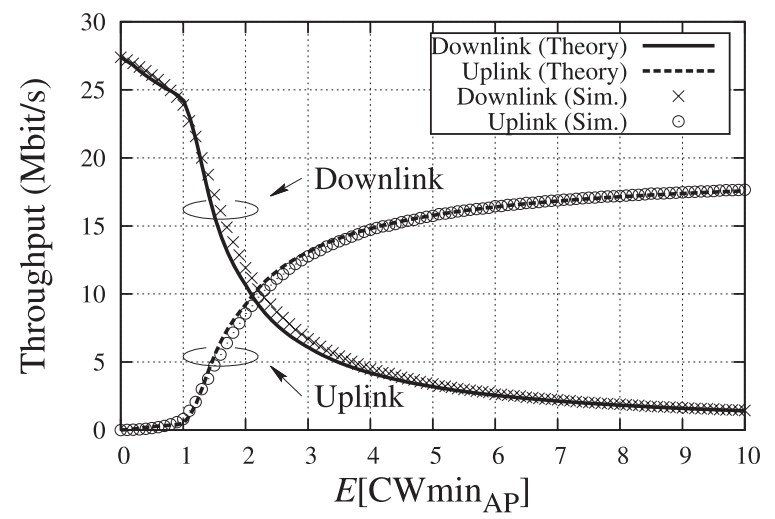

Fig. 6 Throughputs of the proposed scheme (Number of STAs $n: 20$, PHY data rate : $36 \mathrm{Mbit} / \mathrm{s}$ ).

of the throughput. The optimum $E\left[C W \min _{\mathrm{AP}}\right]$ value is obtained by the intersection of uplink and downlink throughput curves in Figs. 5, 6. When we derive the optimum $E\left[C W \min _{\mathrm{AP}}\right]$ value, we can determine the values of $l$ and $\alpha_{\mathrm{HP}}$ by using Eqs. (4) and (3), respectively. The conventional scheme cannot maximize the unidirectional throughput, as shown in Fig. 2, because CWmin ${ }_{\mathrm{AP}}$ value has a nonnegative integer value. In contrast, the proposed VCCC scheme can realize the $E\left[C W \min _{\mathrm{AP}}\right]$ value as a real number and maximize the unidirectional throughput as shown in Fig. 5. The unidirectional throughput is maximized only if the uplink and downlink throughputs are equal. Thus, the proposed VCCC scheme enables accurate control under the constraint that the downlink throughput be exactly equal to the total uplink throughput. The throughputs obtained by the computer simulation are in good agreement with those calculated by the theoretical analysis as shown in Fig. 5. These results reveal that the optimum $E\left[C W \min _{\mathrm{AP}}\right]$ value obtained from the computer simulation and theoretical analysis are 3.55 and 3.5, respectively. On the other hand, in the case of 20 STAs, the difference between the throughputs obtained by the computer simulation and the theoretical analysis become large, as shown in Fig. 6. The reason is described later.

Figure 7 shows the steady-state distribution of the 


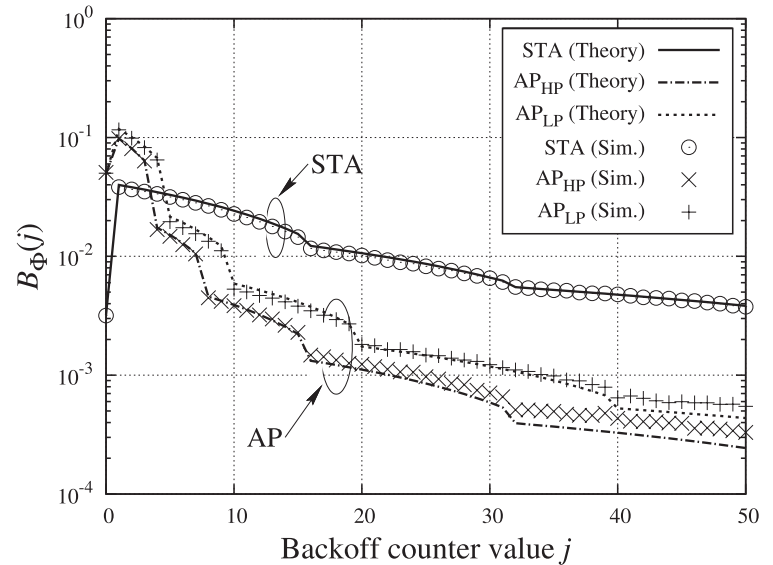

Fig. $7 \quad B_{\Phi}(j)$, steady-state distribution of backoff counter (Number of STAs $n: 10, E\left[C W \min _{\mathrm{AP}}\right]=3.55$, PHY data rate: $\left.36 \mathrm{Mbit} / \mathrm{s}\right)$.

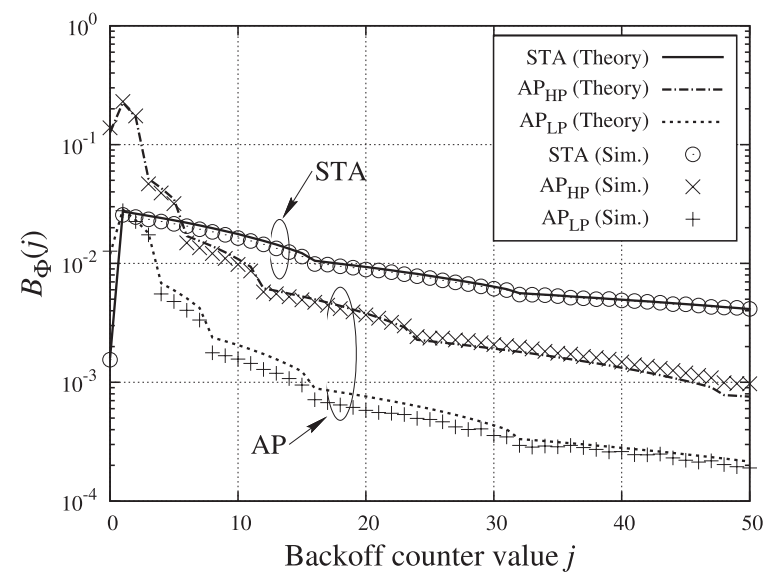

Fig. $8 B_{\Phi}(j)$, steady-state distribution of backoff counter (Number of STAs $n: 20, E\left[C W \min _{\mathrm{AP}}\right]=2.11$, PHY data rate: $36 \mathrm{Mbit} / \mathrm{s}$ ).

$\mathrm{WS}_{\Phi}$ 's backoff counter using the proposed VCCC scheme in the case where 10 STAs exist and $E\left[C W \min _{\mathrm{AP}}\right]$ value is 3.55. Figure 8 shows that in the case where 20 STAs exist and $E\left[C W \min _{\mathrm{AP}}\right]$ value is 2.11 . The numerical values of $B_{\Phi}(j)$ is derived from Eq. (8). The steady-state distribution of backoff counter obtained by the computer simulation are in good agreement with those calculated by the theoretical analysis, as shown in Fig. 7. As the backoff counter value $j$ becomes small, $B_{\Phi}(j)$ has a larger and dominant value. The difference between the theoretical analysis results and the computer simulation results of $B_{\Phi}(j)$ is within $4 \%$ in the case where the backoff counter value is less than three and the number of STAs is 10. In the case where more STAs exist, the difference between the steady-state distributions obtained by the computer simulation and the theoretical analysis becomes large as shown in Fig. 8.

Figures 9 and 10 show the optimum $E\left[C W \min _{\mathrm{AP}}\right]$ values and maximized unidirectional throughputs, respectively. Figure 9 shows that the proposed VCCC scheme can realize the expected value of $\mathrm{CWmin}$ as a real number. Figure 10 shows that the throughput of the proposed scheme is $31 \%$

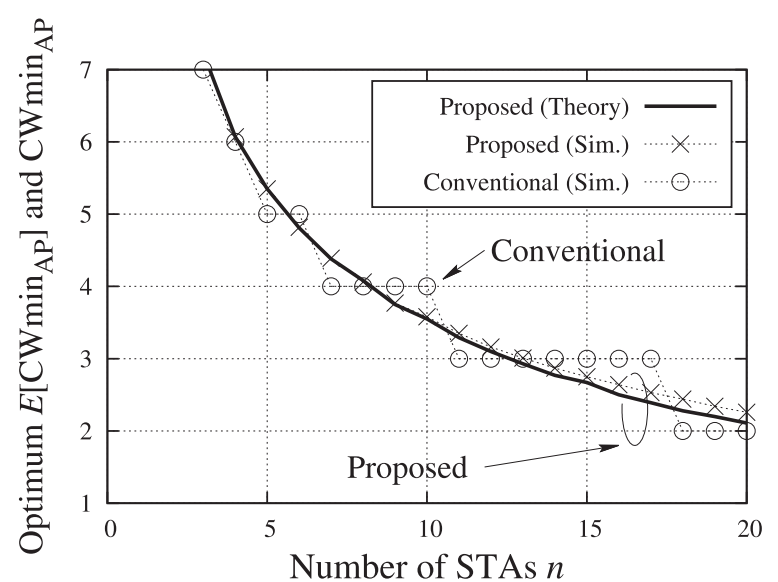

Fig. 9 The optimum $E\left[C W \mathrm{~min}_{\mathrm{AP}}\right]$ of the proposed scheme (PHY data rate : $36 \mathrm{Mbit} / \mathrm{s})$.

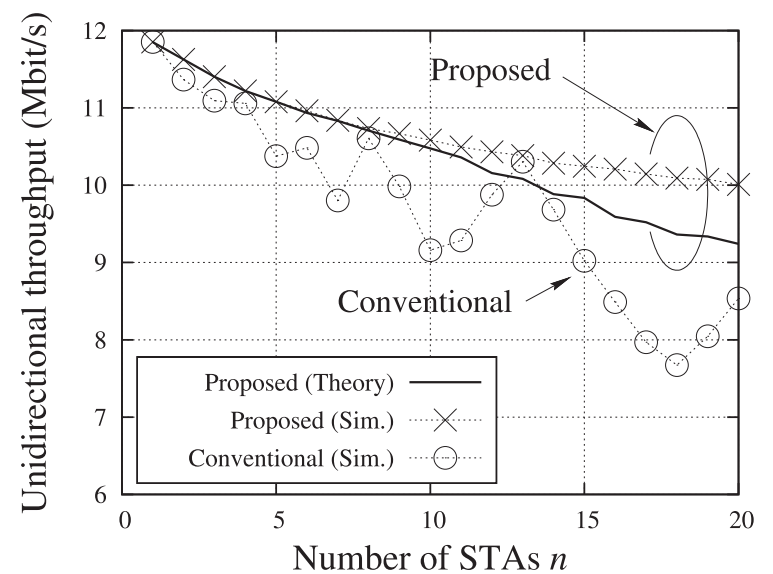

Fig. 10 The unidirectional throughputs of the proposed scheme (PHY data rate : $36 \mathrm{Mbit} / \mathrm{s}$ ).

higher than that of the conventional one when the number of STAs is 18. The difference between theoretical analysis results and computer simulation results of the throughputs is within $1 \%$ when 10 STAs exist. However, the number of STAs increases, the difference between theoretical analysis results and computer simulation results of the throughputs becomes large. The difference is about $8 \%$ when 20 STAs belong to the AP.

The reason is as follows. In the theoretical analysis, it is assumed that $B_{\mathrm{STA}}(j)$ has a constant value at any AP's stages. However it is not practical that $B_{\mathrm{STA}}(j)$ always has a constant value. The value of $B_{\mathrm{STA}}(j)$ depends on the AP's stage. This assumption is the main cause of the difference between the theoretical and the simulation results. Figure 11 shows the probability distribution of STA's backoff counter according to the AP's backoff stage where five STAs exist and $E\left[C W \min _{\mathrm{AP}}\right]$ value is 5.35 . Figure 12 shows those where 20 STAs exist and $E\left[C W m_{\mathrm{AP}}\right]$ value is 2.11 . The difference among the probability distribution of STA's backoff counter becomes large as the number of STAs increases. Thus, theoretical analysis accuracy becomes worse as the 


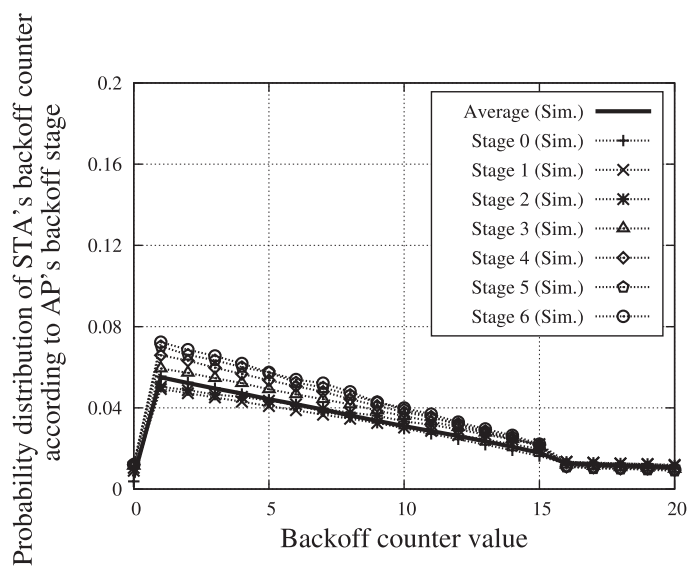

Fig. 11 Probability distributions of STA's backoff counter according to AP's backoff stage (Number of STAs $n: 5, E\left[C W \min _{\mathrm{AP}}\right]=3.55$, PHY data rate : $36 \mathrm{Mbit} / \mathrm{s})$.

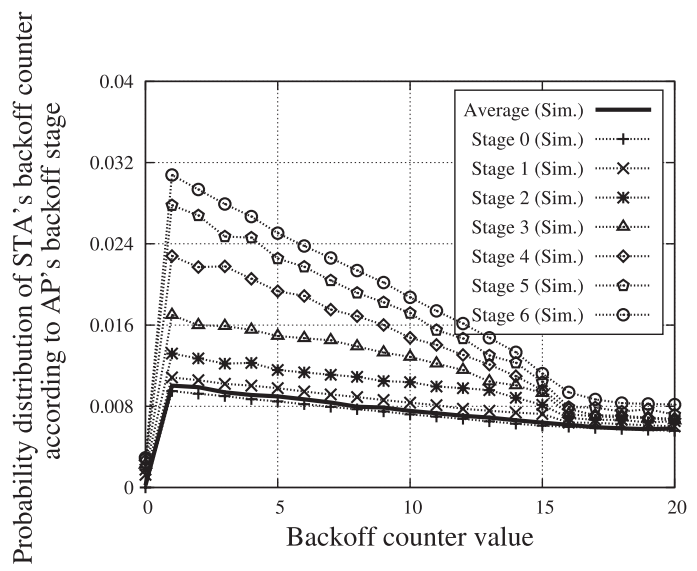

Fig. 12 Probability distributions of STA's backoff counter according to AP's backoff stage (Number of STAs $n: 20, E\left[C W \min _{\mathrm{AP}}\right]=2.11$, PHY data rate : $36 \mathrm{Mbit} / \mathrm{s})$.

number of STAs increases and CWmin $\operatorname{AP}_{\mathrm{P}}$ value decreases.

\subsection{Multi-rate and Unsaturated Traffic Conditions}

The throughput performances for multi-rate networks under saturated or unsaturated traffic conditions are evaluated. The data frame transmission rates of the multi-rate network are $24 \mathrm{Mbit} / \mathrm{s}$ and $36 \mathrm{Mbit} / \mathrm{s}$. Half of the STAs have a data rate of $24 \mathrm{Mbit} / \mathrm{s}$ and the rest of the STAs have a data rate of $36 \mathrm{Mbit} / \mathrm{s}$. Figure 13 shows the unidirectional throughput for a multi-rate network versus the number of STAs under the saturated traffic condition. In addition, the simulation results for multi-rate networks under the unsaturated traffic condition are shown in Fig. 14. This figure shows the unidirectional throughput versus the offered traffic per STA. These results reveal that the proposed VCCC scheme can be successfully applied to not only single-rate networks under saturated traffic conditions but also multi-rate networks under saturated or unsaturated traffic conditions.

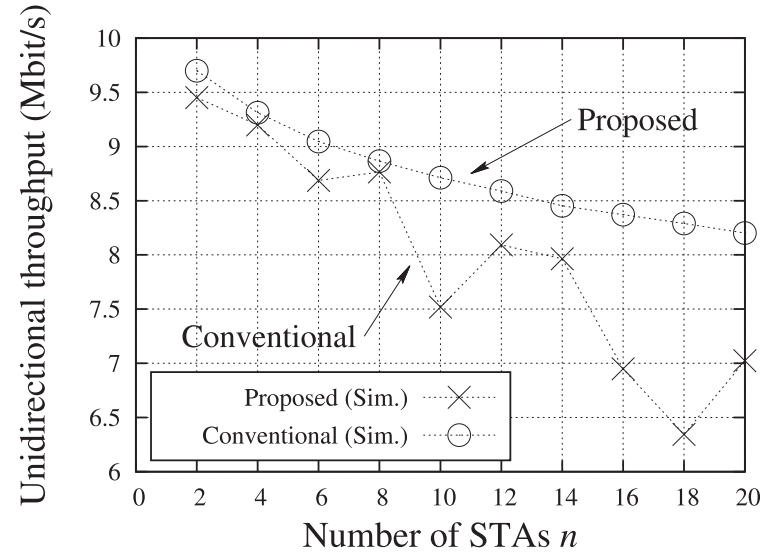

Fig. 13 The unidirectional throughputs of the proposed scheme under the saturated traffic condition (PHY data rate : $36 \mathrm{Mbit} / \mathrm{s}$ and $24 \mathrm{Mbit} / \mathrm{s}$ ).

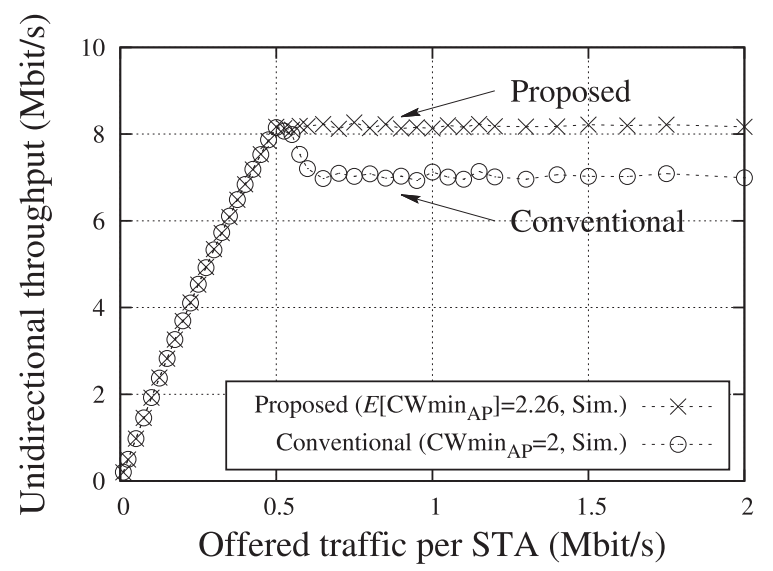

Fig. 14 The unidirectional throughputs vs. the offered traffic per STA (Number of STAs $n: 20$, PHY data rate : $36 \mathrm{Mbit} / \mathrm{s}$ and $24 \mathrm{Mbit} / \mathrm{s}$ ).

\section{Conclusions}

In this paper, we have proposed a new CWmin control method named the VCCC scheme that can realize $E[\mathrm{CWmin}]$ value as a nonnegative real number by changing the CWmin values probabilistically. We have shown that the proposed VCCC scheme can improve the system throughput under the constraint that the downlink throughput be accurately equal to the total uplink throughput. Using a computer simulation, we have estimated the optimized $E[\mathrm{CWmin}]$ value and unidirectional throughput, which is the minimum of the uplink and downlink throughputs in a single-hop WLAN network with the proposed VCCC scheme. We clarified that the unidirectional throughput of the proposed VCCC scheme is $31 \%$ higher than that of the conventional scheme under the saturated traffic condition, when the number of STAs is 18. Moreover, we have proposed the theoretical analysis model for the proposed VCCC scheme and calculated the optimized $E[C W m i n]$ value and unidirectional throughput. The difference between theoretical analysis results and computer simulation results of throughput perfor- 
mances is within $1 \%$ when 10 STAs exist. Furthermore we have revealed that the proposed VCCC scheme can be applied to not only single-rate networks under saturated traffic conditions but also multi-rate networks under saturated or unsaturated traffic conditions.

\section{References}

[1] IEEE Std. 802.11-2007, "Part 11: Wireless LAN medium access control (MAC) and physical layer (PHY) specifications," June 2007.

[2] G. Berger-Sabbatel, A. Duda, O. Gaudoin, M. Heusse, and F. Rousseau, "Fairness and its impact on delay in 802.11 networks," Proc. IEEE GLOBECOM 2004, Dallas, USA, Nov. 29-Dec. 3, 2004.

[3] Y. Fukuda, and Y. Oie, "Unfair and inefficient share of wireless LAN resource among uplink and downlink data traffic and its solution,” IEICE Trans. Commun., vol.E88-B, no.4, pp.1577-1585, April 2005.

[4] D.J. Leith, P. Clifford, and D. Malone, "TCP fairness in $802.11 \mathrm{e}$ WLANs," IEEE Commun. Lett., vol.9, no.11, pp.964-966, Nov. 2005.

[5] X. Jian, G. Ming, X. Ming, and L. Xin, "User fairness of IEEE802.11 WLAN downlink," Proc. 2nd International Conference on Future Computer and Communication (ICFCC 2010), vol.3, pp.V3-148-V3-152, Wuhan, China, May 2010.

[6] B.A.H.S. Abeysekera, T. Matsuda, and T. Takine, "Dynamic contention window control mechanism to achieve fairness between uplink and downlink flows in IEEE 802.11 wireless LANs," IEEE Trans. Wirel. Commun., vol.7, no.9, pp.3517-3525, Sept. 2008.

[7] Y. Sangenya, D. Umehara, M. Morikura, N. Otsuki, and T. Sugiyam, "Novel length aware packet aggregation and coding scheme for multi-hop wireless LANs," Proc. 5th International Conference on Signal Processing and Communication Systems (ICSPCS 2011), Honolulu, Hawaii, USA, Dec. 2011.

[8] I. Tinnirello and G. Bianchi, "Rethinking the IEEE 802.11e EDCA performance modeling methodology," IEEE/ACM Trans. Netw., vol.18, no.2, pp.540-553, April 2010.

[9] MathWorks, http://www.mathworks.com/products/matlab/

[10] QualNet, http://www.scalable-networks.com/

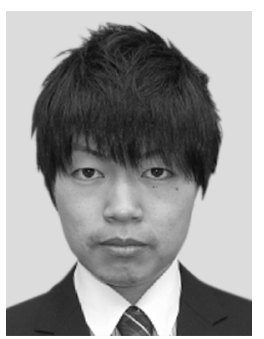

Yuki Sangenya received his B.E. degree from Kyoto University, Japan, in 2011. He is currently a graduate student at Kyoto University, Japan. He has considerable interest in medium access control protocol, especially in the IEEE 802.11 based wireless LAN. He is a student member of IEICE.

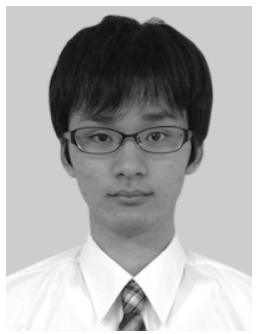

Fumihiro Inoue received his B.E. degree from Kyoto University, Japan, in 2013. $\mathrm{He}$ is currently an M.E. student at Kyoto University, Japan. He has been engaged in Protection Scheme of Wireless Sensor Network against Wireless LAN. He is a student member of IEICE.

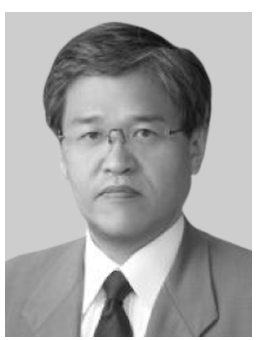

Masahiro Morikura received his B.E., M.E., and Ph.D. degrees in electronics engineering from Kyoto University, Kyoto, Japan in 1979, 1981, and 1991, respectively. $\mathrm{He}$ joined NTT in 1981, where he was engaged in the research and development of TDMA equipment for satellite communications. From 1988 to 1989 , he was with the communications Research Centre, Canada, as a guest scientist. From 1997 to 2002, he was active in the standardization of the IEEE 802.11a based wireless LAN. He received the Paper Award and the Achievement Award from IEICE in 2000 and 2006, respectively. He also received the Education, Culture, Sports, Science and Technology Minister Award in 2007. Dr. Morikura is now a professor in the Graduate School of Informatics, Kyoto University. He is a member of IEEE.

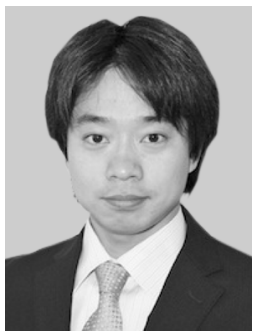

Koji Yamamoto received the B.E. degree in electrical and electronic engineering from Kyoto University in 2002, and the M.E. and Ph.D. degrees in informatics from Kyoto University in 2004 and 2005, respectively. From 2004 to 2005 , he was a research fellow of the Japan Society for the Promotion of Science (JSPS). Since 2005, he has been with the Graduate School of Informatics, Kyoto University, where he is currently an associate professor. From 2008 to 2009 , he was a visiting researcher at Wireless@KTH, Royal Institute of Technology (KTH) in Sweden. His research interests include game theory, spectrum sharing, and M2M networks. He received the PIMRC 2004 Best Student Paper Award in 2004, the Ericsson Young Scientist Award in 2006, and the Young Researcher's Award from the IEICE of Japan in 2008. He is a member of the IEEE.

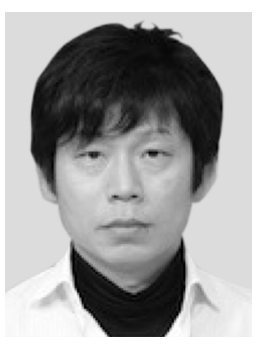

Fusao Nuno received his B.E. degree from Ehime University in 1991 and M.E. degree from Kumamoto University in 1993, respectively in Electrical Engineering. In 1993, he joined NTT and engaged in research and development of portable terminals and base stations for broadband wireless access systems. He is currently a senior research engineer in NTT Access Network Service Systems Laboratories and engaged in the development of the wireless access systems for Wide Area Ubiquitous Network.

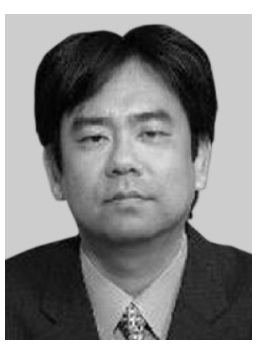

Takatoshi Sugiyama received the B.E., M.E. and Ph.D. degrees from Keio University, Japan in 1987, 1989 and 1998, respectively. Since joining NTT in 1989, he had been engaged in the research of interference compensation, CDMA, MIMO, OFDM technologies for satellite, wireless LAN systems. From 2004 to 2007, he was in Wireless Laboratories of NTT DoCoMo, Inc., where he worked for the research of plug-and-play base stations and wireless mesh networks. He is currently a senior research engineer, supervisor, Group Leader in NTT Access Network Service Systems Laboratories responsible for the research and development of intelligent interference compensation technologies and radio propagation modeling for future wireless communication systems. He received the Young Engineers Award from the IEICE of Japan in 1996. He is a member of the IEEE. 